Association for Information Systems AIS Electronic Library (AISeL)

Wirtschaftsinformatik Proceedings 2003

Wirtschaftsinformatik

September 2003

\title{
MetaWeb: Fachkonzeptionelle Spezifikation WWW-basierter Informationssysteme
}

Jörg Becker

Westfälische Wilhelms-Universität Münster, becker@wi.uni-muenster.de

Christian Brelage

Westfälische Wilhelms-Universität Münster

Michael Thygs

Westfälische Wilhelms-Universität Münster

Karsten Klose

Westfälische Wilhelms-Universität Münster

Follow this and additional works at: http://aisel.aisnet.org/wi2003

\section{Recommended Citation}

Becker, Jörg; Brelage, Christian; Thygs, Michael; and Klose, Karsten, "MetaWeb: Fachkonzeptionelle Spezifikation WWW-basierter Informationssysteme" (2003). Wirtschaftsinformatik Proceedings 2003. 73.

http://aisel.aisnet.org/wi2003/73

This material is brought to you by the Wirtschaftsinformatik at AIS Electronic Library (AISeL). It has been accepted for inclusion in Wirtschaftsinformatik Proceedings 2003 by an authorized administrator of AIS Electronic Library (AISeL). For more information, please contact elibrary@aisnet.org. 
In: Uhr, Wolfgang, Esswein, Werner \& Schoop, Eric (Hg.) 2003. Wirtschaftsinformatik 2003: Medien - Märkte - Mobilität, 2 Bde. Heidelberg: Physica-Verlag

ISBN: 3-7908-0111-9 (Band 1)

ISBN: 3-7908-0116-X (Band 2)

(C) Physica-Verlag Heidelberg 2003 


\title{
MetaWeb: Fachkonzeptionelle Spezifikation WWW-basierter Informationssysteme ${ }^{1}$
}

\author{
Jörg Becker, Christian Brelage, Michael Thygs, Karsten Klose \\ Westfälische Wilhelms-Universität Münster
}

\begin{abstract}
Zusammenfassung: WWW-basierte Informationssysteme werden in Unternehmen $z u$ unterschiedlichsten Zwecken eingesetzt und finden zunehmende Verbreitung. Sowohl die hohe Flexibilität, als auch die Möglichkeit Navigationsstrukturen frei zu gestalten, machen diese Systeme zu einem wertvollen Werkzeug bei der Verwaltung und Nutzung semistrukturierter Daten. Aus Sicht der Wirtschaftsinformatik fehlt es jedoch an geeigneten Methoden zur fachkonzeptionellen Spezifikation $W W W$-basierter Informationssysteme, die deren Besonderheiten Rechnung tragen. Der Aufbau plausibler und akzeptierter Navigationsstrukturen ist von großer Bedeutung für die Akzeptanz und eine effiziente Nutzung des Systems. Die fachkonzeptionelle Spezifikation eines WWW-basierten Informationssystems kann mit Hilfe der Modellierungstechnik MetaWeb durchgeführt werden, die mit Hilfe einer etablierten Methodik zur Entwicklung von Modellierungstechniken konstruiert wird.
\end{abstract}

Schlüsselworte: WWW-basierte Informationssysteme, Modellierung, MetaWeb, Semantic Web, Content Management, Fachkonzeptionelle Spezifikation, Modellierungsmethode

\section{WWW-basierte Informationssysteme als Bestandteil betrieblicher Informationssystem- Architekturen}

Informationssysteme auf Basis von WWW-Techniken sind zu einem festen Bestandteil vieler Informationssystem-Architekturen geworden und werden von Unternehmen zu unterschiedlichsten Zwecken eingesetzt. Neben Internetpräsenzen, die eine komfortable und umfassende Kundenselbstinformation ermöglichen, finden WWW-basierte Informationssysteme auch im Intranet-Bereich, etwa als Bestandteil von Wissensmangement-Systemen, zunehmende Verwendung.

Diese Veröffentlichung wurde gefördert durch das Bundesministerium für Bildung und Forschung, Förderkennzeichen: 01HW0196. 
Eine Definition für WWW-basierte Informationssysteme kann in Anlehnung an den klassischen Informations- und Anwendungssystembegriff gebildet werden. Anwendungssysteme führen automatisierte Aufgaben von Geschäftsprozessen durch und stellen somit den technischen, computergestützten Teil eines Informationssystems dar [vgl. Seib01, S. 46]. Informationssysteme sind umfangreicher zu verstehen, da es sich um Mensch-Computer oder sozio-technische Systeme im jeweiligen individuellen organisatorischen Kontext handelt [vgl. Krcm03, S, 25ff; FeSi01, S. 8f; Teub9, S. 26f; BeSc96, S. 9f;], bei deren Gestaltung neben der technischen Komponente organisatorische Aspekte wie „betriebliche Aufgaben/ Prozesse“, „Menschen/ Personen“ und „Organisationseinheiten“ berücksichtigt werden [vgl. Seib01, S. 47].

Im Rahmen dieses Beitrages werden unter WWW-basierten Informationssystemen jene Informationssysteme verstanden, deren Anwendungssysteme auf eine einfache Navigation in großen Datenbeständen auf Basis grundlegender WWWTechniken wie HTML, XML, HTTP etc. ausgerichtet sind. Umfangreiche Internetanwendungen wie Web-Shops, Web-Frontends betrieblicher Standardsoftware, die komplexe Ablauflogik implementieren werden im Folgenden nicht betrachtet.

Die hier angesprochenen WWW-basierten Informationssysteme erfreuen sich großer Popularität. Als Ursachen lassen sich u. a. folgende Gründe anführen:

- Komplexe Navigationsstrukturen und hohe Flexibilität: Navigationsstrukturen über den Datenbestand lassen sich einfach und schnell (gegebenenfalls dynamisch) erzeugen. Dadurch können personalisierte Navigationsstrukturen erzeugt werden, wodurch Akzeptanz und Effizienz des Informationssystems gesteigert werden. Ferner ermöglichen offene Standards und Dokumentenformate eine hohe Flexibilität bei Gestaltung, Implementierung und Integration eines WWW-basierten Informationssystems.

- Geringe Kosten: Die notwendige Rechenleistung für Server und Clients ist in der Regel zu vernachlässigen. Ferner ist die benötigte Software (Browser und Web-Server) preiswert oder gar kostenlos erhältlich und für nahezu alle Plattformen verfügbar. Zudem sind Benutzer in der Regel mit Browsern vertraut, so dass der Schulungsaufwand gering gehalten werden kann.

Trotz dieser Vorteile weisen WWW-basierte Informationssysteme Nachteile auf, die einer weiteren Verbreitung und intensiveren Nutzung entgegenstehen:

- Die großen Datenmengen im Internet oder Intranet erschweren zunehmend die effiziente Suche nach den gewünschten Informationen.

- Die Daten werden üblicherweise nicht mit Metadaten annotiert. Dies erschwert sowohl die automatische Verarbeitung als auch die sinnvolle Interpretation der Daten durch den Benutzer.

- Für den Aufbau geeigneter Navigationsstrukturen, die eine effiziente Suche ermöglichen, ist eine geeignete Verschlagwortung der enthaltenen Daten er- 
forderlich. Zu diesem Zweck werden Schlagworte benötigt, die für alle Benutzer des Systems die gleiche Bedeutung haben. Insbesondere in großen Organisationen ist diese Verschlagwortung problematisch (Synonyme, Homonyme, etc.) und uneinheitlich.

- Navigationsstrukturen über die Daten werden momentan üblicherweise aus rein technischen Gesichtspunkten oder über einfache Hierarchien gebildet. Für die Navigation im Datenbestand ist neben der technischen Navigation auch eine semantische Navigation über die Bedeutung und Bedeutungszusammenhänge der enthaltenen Dokumente notwendig. Eine einfache Hierarchisierung der Daten ist nicht ausreichend, da verschiedene Benutzer häufig unterschiedliche Suchwege zu gleichen Daten benötigen bzw. präferieren. So stößt etwa die Ablage von Dokumenten im hierarchischen Dateisystem auf Grund von Personalwechseln oder Umstrukturierungen im Unternehmen an ihre Grenzen. Ferner lassen sich viele Dokumente nicht immer eindeutig einer Kategorie zuordnen (bspw. ein Artikel eines Nachrichtendienstes über zwei Produkte eines Unternehmens).

Generell können WWW-basierte Informationssysteme zur Speicherung und Verwaltung semistrukturierter Daten verwendet werden, deren Eigenschaften (statische und dynamische Irregularität und fehlende Schemainformationen [vgl. BeLa03 S. 4f]) maßgeblich für die genannten Defizite heutiger Systeme verantwortlich sind.

In letzter Zeit wird verstärkt an der Behebung dieser Unzulänglichkeiten gearbeitet. Unter dem Schlagwort ,Semantic Web' [vgl. Ber ${ }^{+} 01$, o. S.] wird von dem Standardisierungsgremium World Wide Web Consortium (siehe http://www.w3.org) die Annotation von Daten mit Metadaten gefordert. Technische Grundlage sind das Resource Description Framework [LaSw99,o. S.; $\mathrm{Can}^{+} 01$, S. 6ff] oder Web Ontologies [Smi ${ }^{+}$02, o. S.]. Gemein ist diesen Ansätzen, dass durch die Annotation der Daten mit Metadaten die Interpretier- und Auffindbarkeit der Daten durch den Benutzer verbessert, und durch die Beschreibung der Dokumente bzw. Daten durch eindeutige Begriffssysteme (Ontologien) verständliche und allgemein akzeptierte Navigationsstrukturen erzeugt werden sollen. Zudem soll auf diesem Wege die automatische Verarbeitung der Daten (etwa durch Agenten) ermöglicht werden.

Die Forschung in diesem Bereich zeichnet sich derzeit durch eine hohe Implementierungsnähe aus. In der Vergangenheit gab es jedoch verschiedene Ansätze, um den Besonderheiten WWW-basierter Informationssysteme auch auf fachkonzeptioneller Ebene im Rahmen spezieller Modellierungsmethoden Rechnung zu tragen. Zu nennen sind hier HDM [Gar'93, S. 1ff], Dexter [HaSc90, S. 95ff], OOHDM [Sch ${ }^{+}$96, S. 116ff], W3DT [BiNu96, S. 328ff], RMM [Isa ${ }^{+} 95$, S. 34ff], Araneus [Meri+03, S. 49ff] und WebML [Cer ${ }^{+} 00$, S. 137ff]. Eine kritische Untersuchung einiger dieser Methoden findet sich in [Ehle03, S. 74ff]. Die jüngeren der genannten Methoden (OOHDM, W3DT, Araneus, WebML) verfolgen einen CASE- 
Ansatz und bieten teilweise sehr umfangreiche und komplexe Modellierungsmöglichkeiten, die wie im Falle WebML oder Araneus teilweise auch in speziellen Modellierungstools umgesetzt wurden.

Anforderungen an eine Modellierungsmethode zu fachkonzeptionellen Spezifikation [vgl. Sche98, S. 14f.; BeSc96, S.19f.] können in Anlehnung an die Grundsätze ordnungsgemäßer Modellierung (GoM) $\left[\mathrm{Bec}^{+} 95\right.$, S. 335ff] gestellt werden:

- Verständlichkeit und leichte Kommunizierbarkeit: Die Technik muss die Kommunikation zwischen Fachanwender und Modellierer effizient unterstützen. Folglich ist ein geeignetes Abstraktionsniveau zu wählen und die Komplexität der Methode in akzeptablen Grenzen zu halten. In der Phase der fachkonzeptionellen Spezifikation sollte so weit wie möglich von implementierungsrelevanten Details abstrahiert werden, um die erstellten Modelle auch für technisch weniger versierte Fachanwender verständlich zu halten, da die Explikation betriebswirtschaftlicher Anforderungen und nicht deren technische Umsetzung im Vordergrund steht. Vereinfacht ausgedrückt wird in dieser Phase festgelegt, welche Inhalte an welcher Stelle des Navigationsraumes zu platzieren sind und wie der Navigationsraum selbst gestaltet ist. Fragen der Implementierung (etwa einer Skriptsprache, der Speicherung im File-System oder in einer Datenbank etc.) werden in dieser Phase nicht berücksichtigt.

- Navigation: Bei der Gestaltung WWW-basierter Informationssysteme steht die Konzeption der Navigationsstrukturen im Vordergrund da diese wesentlich die Produktivität und Akzeptanz des Systems beeinflussen. Eine geeignete Modellierungsmethode muss deren einfache Darstellung effizient unterstützen und Vorschläge zur plausiblen Ableitung der Navigationsstrukturen unterbreiten.

- Formale Korrektheit und systematischer Aufbau: Eine Modellierungstechnik muss intersubjektiv nachprüfbar und logisch konstruiert sein. Die elementaren Begriffe und Relationen der Modell- bzw. Sprachelemente müssen präzise expliziert und vereinbart werden, damit die Bedeutung der Sprachelemente eindeutig nachvollzogen werden kann.

- Formalisierungsgrad: Das mit der Technik erstellte Modell hinreichend formal sein, um den Entwicklungsprozess des Informationssystems zu unterstützen und gegebenenfalls Entwicklungsschritte (teilweise) zu automatisieren.

Die genannten Modellierungsmethoden erfüllen diese Anforderungen nur teilweise. Die Komplexität der Modellierungsmöglichkeiten, die eine teilautomatische Erzeugung der WWW-Anwendung ermöglicht, ist in der Konzeptionsphase einer leichten Kommunizierbarkeit mit Fachanwendern nicht zuträglich. Beispielsweise ist das Navigational Class Schema von OOHDM [vgl. Schw ${ }^{+}$66, S. 121f] bereits sehr detailliert (viele Symbole) und für Fachanwender, die mit der Methode weniger vertraut sind, nur schwer nachvollziehbar. Ähnliches gilt für WebML, die sehr viele Symbole und Teilmodelle umfasst. Viele Sprachelemente dieser Methoden werden erst auf der Ebene der DV-Konzeption benötigt. Die Methoden fokussie- 
ren ferner überwiegend den technischen Teil eines WWW-basierten Informationssystems. Die Verknüpfung der Inhalte mit dem bestehenden Informationsraum in den Köpfen der Mitarbeiter steht nicht im Vordergrund.

Aus Sicht der Wirtschaftsinformatik ist daher im Bereich der fachkonzeptionellen Spezifikation WWW-basierter Informationssysteme ein Theoriedefizit zu konstatieren, zu dessen Lösung in diesem Artikel ein Vorschlag in Form der Modellierungstechnik MetaWeb unterbreitet wird. Der Fokus dieses Artikels liegt auf der Verwendung WWW-basierter Systeme im Intranet-Bereich (etwa als Bestandteil von Wissensmanagement-Systemen), ohne jedoch die Anwendbarkeit der zu entwickelnden Methode MetaWeb auf diesen Bereich zu beschränken.

In den folgenden Kapiteln wird die Modellierungstechnik MetaWeb schrittweise konstruiert. Zu diesem Zweck folgt zunächst ein Überblick über die zu Grunde liegende Forschungsmethodik in Kapitel zwei. In Kapitel drei wird die Orthosprache der Modellierungstechnik MetaWeb definiert und mit Hilfe eines Metamodells in ERM-Notation veranschaulicht. Ein Vorschlag für eine mögliche Notation sowie diverse Beispiele für Navigationsstrukturen, die mit dieser Methode spezifiziert werden können, folgt in Kapitel vier. Abschließend folgt ein Ausblick auf weitere Arbeiten sowie offenen Forschungsbedarf.

\section{Grundlagen der Methodenentwicklung}

\subsection{Methodenentwicklung als Kerndisziplin der Wirtschaftsinformatik}

Die Entwicklung von Informationssystemen mit Hilfe fachkonzeptioneller Modelle, und damit die Entwicklung geeigneter Modellierungsmethoden für den jeweiligen Aufgabenkomplex, ist eine Kerndisziplin der Wirtschaftsinformatik [vgl. $\mathrm{Bec}^{+}$01, S. 3]. Bei der Modellierung eines Informationssystems, ist vom Modellierer jeweils die Entscheidung für eine (oder wenige) Modellierungstechniken zu treffen. Diese Entscheidung ist auf Grund der Vielzahl und Verschiedenartigkeit der Techniken (bspw. die möglichen Varianten zur Modellierung der Datensicht) und der Komplexität der Aufgabestellung nicht trivial. Erschwert wird die Entscheidung für oder gegen eine spezielle Technik durch die geringe Vergleichbarkeit der Techniken untereinander.

Alternativ zur Auswahl einer existierenden Technik, kann versucht werden, für die gegebene Aufgabenstellung eine neue Modellierungstechnik zu konstruieren. Durch die Neukonstruktion ergibt sich die Möglichkeit, auf technische Neuerungen zu reagieren und diese bereits im Rahmen der fachkonzeptionellen Spezifika- 
tion zu berücksichtigen. Der Modellierer nimmt in diesem Fall die Rolle des $M e$ thodenkonstukteurs $\left[\mathrm{Bec}^{+} 01\right.$, S. 4] ein.

Um die genannten Anforderungen an eine Modellierungsmethode zu erfüllen, ist, wie für die Anwendung einer Methode, auch für deren Entwicklung ein methodisches, theoretisch fundiertes Vorgehen unerlässlich. Daher werden im folgenden Kapitel die methodischen Grundlagen der Methodenentwicklung kurz beleuchtet.

\subsection{Terminologische und methodische Grundlagen der Methodenentwicklung}

Trotz der Bedeutung der Methodenanwendung und -entwicklung für die Wirtschaftsinformatik, sind die zentralen Begriffe (Methode, Methodik, Technik, Sprache etc.) aus diesem Aufgabengebiet in der Literatur nicht eindeutig belegt, und sollen daher überblicksartig erarbeitet werden [vgl. $\mathrm{Bec}^{+} 01$, S. 5ff], um in den folgenden Kapiteln eine einheitliche Terminologie zu Grunde legen zu können.

Die Verwendung von Modellen und theoretisch fundierten Methoden zur strukturierten Problemlösung ist den Ingenieursdisziplinen entlehnt. Die Verwandtschaft mit den Ingenieursdisziplinen kommt auch in dem Begriff Information System Engineering, der den schöpferischen Charakter der Tätigkeit betont, zum Ausdruck. Zur Erarbeitung einer Problemlösung werden in beiden Disziplinen strukturierte Lösungsvorschläge in Form von Methoden unterbreitet.

Im Allgemeinen wird unter einer Methode ein auf einem Regelsystem aufbauendes Verfahren verstanden, das Lösungen für einen bestimmten Typ von Problemen liefert [Teub99, S. 93]. Um den genannten Anforderungen an Modellierungstechniken Rechnung zu tragen, bedienen sich diese üblicherweise formaler Sprachen und grafischer Notationen. Die im Rahmen der Informationssystementwicklung konstruierten Modelle weisen in Abhängigkeit von der jeweiligen Beschreibungsebene (Fachkonzept, DV-Konzept, Implementierung) einen unterschiedlich hohen Abstraktions- bzw. Formalisierungsgrad auf. Gemäß der Zielsetzung dieses Artikels, werden an dieser Stelle Modelle auf fachkonzeptioneller Ebene konstruiert, an die, ergänzend zu Absatz 2.1, die Anforderung der einfachen, gegebenenfalls automatischen Überführbarkeit in implementierungsnähere Modelle gestellt werden kann.

Eine Modellierungstechnik stellt einen operationalisierten Ansatz zur Modellerstellung dar [vgl. $\left.\mathrm{Bec}^{+} 01, \mathrm{~S} .8 \mathrm{f}\right]$. Die Modellierungstechnik besteht aus einer Handlungsanleitung und der Modellsprache. Die Handlungsanleitung legt Regeln fest, wie die in der Sprache festgelegten sprachlichen Mittel zu verwenden sind. Die Modellsprache der Modellierungstechnik besteht aus der Orthosprache und einer Notation. Die Orthosprache definiert die Sprachelemente und ihre Beziehungen eindeutig und zirkelfrei. Auf diese Weise wird die Bedeutung der Sprachelemente und ihrer Beziehungen normiert, so dass Modellersteller und -anwender 
eine eindeutige domänenbezogene Grundlage für den Aufbau bzw. die Nutzung der erstellten Modelle haben. Methodische Grundlage für die Konstruktion der Orthosprache bildet der sprachkritische Ansatz von Kamlah und Lorenzen [KaLo73]. Zur Formalisierung der Orthosprache haben sich sprachbasierte Metamodelle bewährt [Strah96, S. 9ff; Holt99, S. 11f]. Aus Sicht des zu beschreibenden Sachverhaltes ist die Orthosprache ein sprachbasiertes Metamodell, da in der Orthosprache die Elemente für die Modellebene spezifiziert werden. Durch diese Darstellung wird der fachsprachliche Aspekt der Sprache expliziert.

Die Notation ordnet schließlich den in der Orthosprache definierten Sprachelementen sowie deren Beziehungen geeignete Repräsentationsformen zu (in der Regel in grafischer Form). Die soeben eingeführten grundlegenden Begriffe werden in der folgenden Abbildung veranschaulicht.

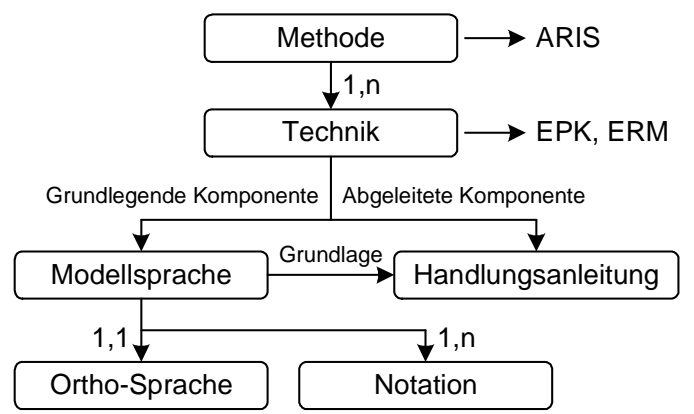

Abbildung 1: Komposition von Methoden [vgl. $\mathrm{Bec}^{+} 01$, S. 7f]

Auf Basis der soeben eingeführten Terminologie kann nun eine Technik zur fachkonzeptionellen Spezifikation von Informationssystemen entwickelt werden. $\mathrm{Zu}$ diesem Zweck wird zunächst die Orthosprache definiert. Anschließend werden den Sprachelementen grafische Repräsentationsformen zugeordnet mit deren Hilfe formale Modelle erstellt werden können.

\section{MetaWeb: Fachkonzeptionelle Spezifikation WWW-basierter Informationssysteme}

In der Forschungsmethode ist die Definition der Orthosprache der Ausgangspunkt für die Konstruktion der Modellierungsmethode. In der Orthosprache werden die Begrifflichkeiten und deren Relationen definiert. Diese konstruierte Sprache wird durch methodische Rekonstruktion, der in dem Anwendungsbereich der WWWbasierten Informationssysteme benutzten natürlichen Sprache, gewonnen [Lehm99, o. S.]. Die verwendete Methode der Metamodell-basierten Modellierung von Informationssystemen basiert auf Holten [Holt99, S. 12ff], der diese für die 
Modellierung von Führungsinformationssystemen entwickelt hat. Zur Darstellung (vgl. Abbildung 3) der statischen Beziehungen der Begrifflichkeiten wird das Entity-Relationship-Modell [vgl. Chen76] in der Erweiterung um Kardinalitäten verwendet [BeSc96, S. 31-37].

Ausgangspunkt für die Erstellung von Content ist das atomare Element. Dieses stellt die kleinste Informationseinheit (Dateneinheit) dar, die in dem System verwaltet und gepflegt werden soll. Die atomaren Elemente können Texte, Bilder oder Videos sein. Attribute sind Eigenschaften und Metadaten, die in Verbindung mit atomaren Elementen den elementaren Entitytyp Content konstituieren. Attribute sind z. B.: Schlagworte, Überschriften, Autorendaten, Erstellungsdatum. Diese stehen innerhalb des Systems fest, und können interpretiert werden (gegebenenfalls automatisch). Attribute können in Attributgruppen zusammengefasst werden, wobei den Attributgruppen mindestens ein Attribut zugeordnet wird. Über diese Zuordnung können Gruppen gebildet werden, die im Weiteren einen Content klassifizieren. Beispielsweise können die Attribute Überschrift, Erscheinungsdatum, Autor, Kurztext, Schlagworte zu einer Attributgruppe Pressetext zusammengefasst werden. Die Vererbung der Attributgruppen unterstützt die Wiederverwendung von Attributgruppen und ermöglicht die Bündelung von Attributen zu festen Einheiten. Die Attribute Vorname, Nachname, Titel können mit Hilfe der Vererbung zu der Attributgruppe Autor zusammengefasst werden, so dass dieses Konstrukt in verschiedenen Attributgruppen wieder verwendet werden kann. Die Vererbung ist dabei als strikte Hierarchie modelliert, um einen konsistenten Aufbau der vererbten Attributgruppen sicherzustellen.

Der Content ergibt sich aus der Kombination von atomaren Elementen und Attributgruppen. Diese Kombination führt dazu, dass die Rohdaten (atomare Elemente) automatisch verarbeitet werden können. Durch die Zuordnung eines Publikationszeitraums (Attribut) zu einem Text (atomares Element) wird z. B. ein Content erzeugt, der von dem Informationssystem nur im gegebenen Publikationszeitraum angezeigt wird. Das Zurückziehen des Textes könnte automatisch erfolgen. Dies resultiert aus der Zuordnung von Attributgruppen, die im Kontext des Informationssystems eine Bedeutung besitzen. Damit wird die Grundlage für eine automatische Weiterverarbeitung gelegt. Die Contentstruktur stellt verschiedene selbstständige Contents zu umfangreicheren Contents zusammen (z. B. die Zusammensetzung mehrerer Kapitel zu einem Aufsatz).

Navigationsknoten und deren Struktur bilden eine grundlegende Navigationskomponente des Informationssystems. Navigationsknoten können beliebig miteinander verknüpft werden und bilden die Basis aller Verknüpfungen (Links) in dem System. Eine explizite Modellierung der Navigationsknotenstruktur ist nicht erforderlich, da diese automatisch angelegt und verwaltet werden können. Durch die eindeutige Zuweisung eines Navigationsknotens zu einem Content, wird jedem Content (mindestens) eine eindeutige Adresse (URL) zugewiesen, über die auf den Content zugegriffen werden kann. Im Gegensatz zu den später zu erläuternden Referenzobjekten implizieren Navigationsknoten keinerlei Semantik. 
Zur Darstellung des Layouts des Contents werden Layoutregeln benötigt, die über die Layoutregelgruppe zusammengefasst werden können. Die Vererbung ermöglicht eine hierarchische Bildung von Layoutstrukturen (vgl. Vererbung bei Attributen). Dieses Konstrukt wird benötigt, um Cascading Style Sheet (CSS)Spezifikationen $\left[\mathrm{Bos}^{+} 98\right.$, o. S.] abzubilden. Über den Relationshiptyp Co-LRGZO können einem Content unterschiedliche Layouts zugewiesen werden.

Die bisher beschriebenen Elemente der Modellierungstechnik beschreiben das WWW-basierte Anwendungssystem, da bisher nur auf die Erzeugung und Speicherung des verwalteten Contents eingegangen wurde. Um die eingangs erwähnte Forderung nach plausiblen und semantisch bedeutsamen Navigationsstrukturen erfüllen zu können, wird im Folgenden die Zuordnung von Inhalten zu realen oder gedachten Objekten beschrieben. Auf diese Weise soll Content mit dem (gedachten) Informationsraum der Benutzer des Informationssystems verbunden werden. Darauf aufbauend können Strukturen erzeugt werden, die für alle Benutzer des Systems die gleiche Bedeutung implizieren. Das Konzept der Modellierung des Informationsraumes orientiert sich an der MetaMIS-Methode [vgl. Holt99; Holt01], die vergleichbare Konstrukte für die fachkonzeptionelle Spezifikation von Information Warehouse Projekten vorsieht.

Dieser Informationsraum wird mit Hilfe der Dimensionen modelliert, die einen Raum der sachlich-zeitlichen Struktur des relevanten Unternehmenskontextes aufspannen $\left[\operatorname{Codd}^{+} 93\right.$, S. 12ff.]. Eine ausführliche Beschreibung und wissenschaftlichen Fundierung des Dimensionskonzepts findet sich bei Holten [Holt99, S. 82f.]. Die Dimensionen ergeben sich aus ,der Wahrnehmung und zweckgeleiteten Konstruktion des Modellerstellers" [Holt99, S. 82]. Eine typische Dimension ist bspw. die Zeit, die durch einen Zeitstrahl repräsentiert wird. Da im WWWbasierten Informationssystem qualitative Kriterien relevant sind, kann eine weitere Dimension die Produktpalette eines Unternehmens sein. In der Abbildung 2 ist bspw. ein Informationsraum mit den Dimensionen Region, Zeit und Produkt abgebildet. Die Dimensionen bieten eine erste Möglichkeit entlang der Dimensionsausprägungen zu navigieren (Im Beispiel: von 2003 nach 2004 oder von Milch zu Brot).

In den Informationsraum werden die Referenzobjekte, die Objekte der Vorstellungs- oder Realwelt repräsentieren, eingeordnet. Dieses Konstrukt wurde in Anlehnung an die Bezugsobjekte von Holten gebildet [vgl. Holt99, S. 78ff]. Die Referenzobjekte stellen zeitlich und sachlich abzugrenzende Untersuchungsobjekte des Anwendungskontextes dar. Zusammen mit den Referenzobjektstrukturen dienen sie als Container für sämtliche im Kontext des WWW-basierten Anwendungssystems relevanten Objekte und deren Strukturen. Es wird ermöglicht, die stabilen, etablierten und bekannten Strukturen der Referenzobjekte mit den Contents eines WWW-basierten Informationssystems zu verbinden. Beispiele für Referenzobjekte und Referenzobjektstrukturen sind:

- Benutzer und Benutzerrollen für die Zugriffssteuerung 
- Mitarbeiter und Organigramme

- Kunden und Verkaufsregionen

- Warengruppen und Warengruppenhierarchie

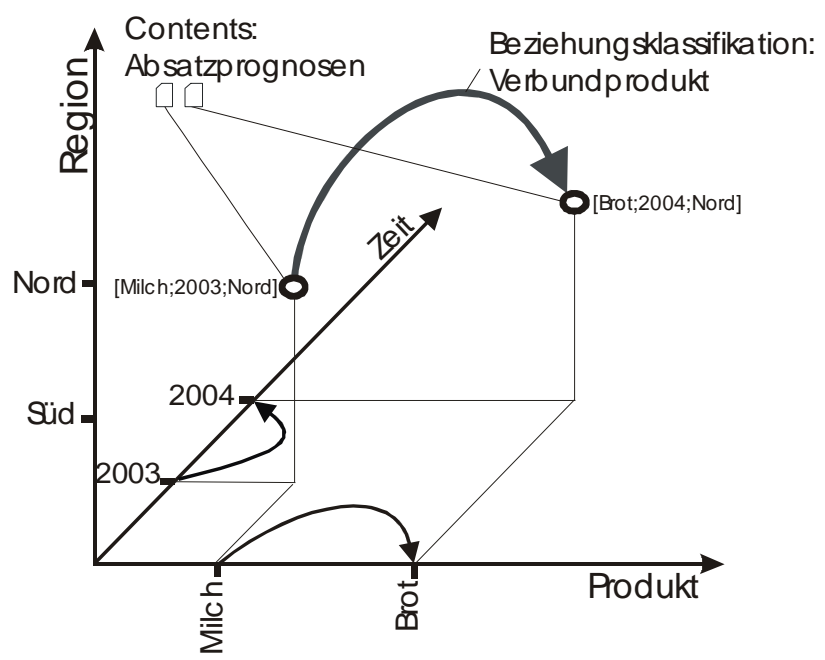

Abbildung 2: Informationsraum

Die Referenzobjekte und Referenzobjektstrukturen werden auf der Metaebene nicht explizit modelliert, da es sich bei der Definition um eine Aufgabe bei der Modellierung auf Typebene handelt, d.h. eine Aufgabe im Modellerstellungsprozess ist die Identifikation und Abbildung der existierenden Referenzobjekte. Allgemein ist die Erstellung von Referenzobjekten und Referenzobjektstrukturen einer der wichtigsten und anspruchvollsten Aufgaben bei der Erstellung eines Informationssystems. Referenzobjekte stellen Netze wichtiger Begrifflichkeiten dar, die im Vergleich zu den Navigationsknoten auf einem semantischen Zusammenhang beruhen. Referenzobjekte können in Dimensions- und KombinierteReferenzobjekte spezialisiert werden. In dem Entitytyp DimensionsReferenzobjekt befinden sich Referenzobjekte, die eindeutig einer Dimension zugeordnet werden. Ein Dimensions-Referenzobjekt ist in der Abbildung 2 ein Objekt, das einem Punkt auf den Koordinatenachsen zugeordnet werden kann. Über die Dimensions-RO-Hierarchie werden die Elemente einer Dimension zusammengefasst und in einer Hierarchie geordnet. In der Anwendung in einem WWW-basierten Anwendungssystem würde damit impliziert, dass alle Contents, die mit den Referenzobjekten verknüpft sind, zusammengefasst werden. Das Kombinierte-Referenzobjekt zeichnet sich dadurch aus, dass es mehr als einer Dimension zugeordnet wird. Das kombinierte Referenzobjekt besteht aus einem nTupel an Dimensionszuordnungen. In der Abbildung 2 sind zwei KombinierteReferenzobjekte [Milch;2003;Nord] und [Brot; 2004;Nord] dargestellt. 
Der Entitytyp Dimensionsausschnitt stellt eine Sammlung von DimensionsReferenzobjekten dar und ermöglicht es, zusammen mit den Dimensionsausschnitts-Kombinationen einen beliebigen Teilraum aus dem durch die Dimensionen aufgespannten Raum auszuschneiden. Die relevanten Contents des Ausschnitts können damit beliebigen Teilräumen zugeordnet werden.

Die Beziehungsklassifikation wird zur Beschreibung der Beziehungsart von $\mathrm{Zu}-$ ordnungen verwendet, d.h. die Beziehungen zweier Entitytypen kann spezifiziert werden. Eine solche Zuordnung wird in der Contentstruktur, Referenzobjektstruktur und in der URL hergestellt. In den Struktur und Verknüpfungen werden zwei gleichwertige Entitäten miteinander verbunden. Die Art der Beziehung wie ,ist untergeordnet“, ,,ist synonym“, ,ist vergleichbar“, „,ist Einleitung“ o.ä kann mit Hilfe der Beziehungsklassifikation festgelegt werden. In der Kombination mit einem Referenzobjekt (BK-RO) werden klassifizierende Eigenschaften den Referenzobjekten zugeordnet. Beispielsweise kann das Referenzobjekt ein Mitarbeiter sein und die Beziehungsklassifikation ist ,hat keinen Zugriff'. Diese Kombination kann verwendet werden, um dem Mitarbeiter den Zugriff auf bestimmte URLs zu verweigern, in dem die entsprechende Entität aus dem Relationshiptyp BK-RO einer URL zugeordnet wird. Ein weiteres Beispiel befindet sich in Abbildung 2, in dem eine Beziehung zwischen den beiden Referenzobjekten dargestellt wird, die mit der Wertung ,sind Verbundprodukte“ klassifiziert ist. Es bleibt anzumerken, dass die Beziehung selber in dem Relationshiptyp Referenzobjektstruktur erfasst wird.

Im Vorangegangenen wurden bereits die Verknüpfung zwischen dem WWWbasierten Anwendungssystem und dem Informationsraum des Unternehmenskontextes angedeutet. Die explizite Zuordnung zwischen den Elementen wird mit dem Relationshiptyp CO-RO-ZO zwischen dem Content und dem Referenzobjekt hergestellt. Jedem Content muss mindestens ein Referenzobjekt zugeordnet werden, damit die Verbindung zwischen dem Anwendungssystem und dem Informationssystem aufrechterhalten wird. An dieser Stelle werden die Referenzobjekte des Unternehmenskontextes mit den Contents des WWW-basierten Anwendungssystems verbunden. Die folgende Grafik fasst die Elemente der Sprache MetaWeb in einem sprachbasierten Metamodell in ERM-Notation zusammen. 


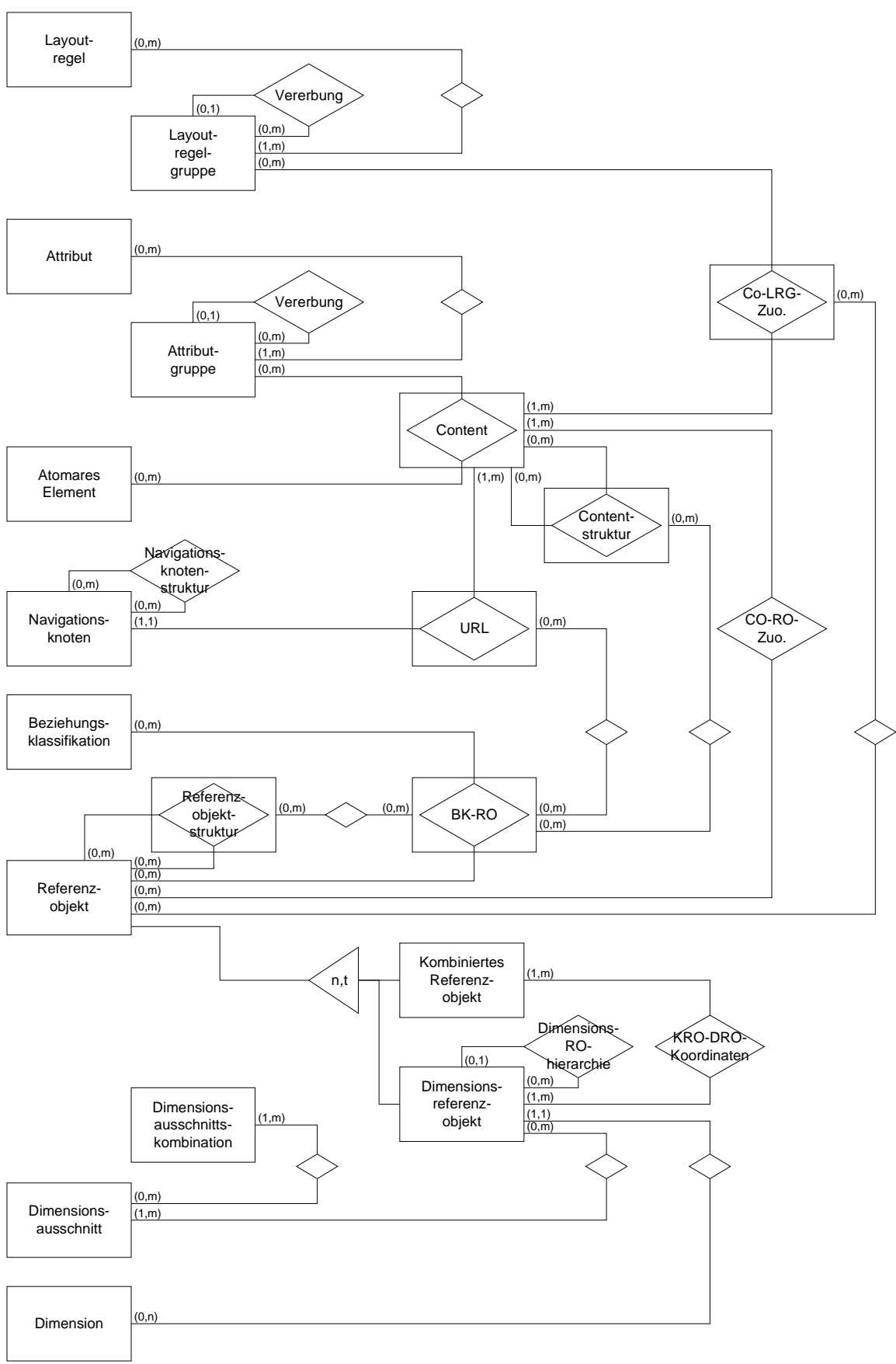

Abbildung 3: Sprachbasiertes Metamodell der Technik MetaWeb 


\section{MetaWeb: Notation und Beispiele}

\subsection{Notation der Technik MetaWeb}

Basierend auf den Ausführungen in Kapitel 2.2 und 3 können den Elementen der Orthosprache nun grafische Repräsentationsformen zugeordnet werden. Aus Gründen der Vereinfachung, werden an dieser Stelle nicht alle in der Orthosprache definierten Sprachelemente verwendet, sondern nur diejenigen expliziert, die in den folgenden Beispielen Verwendung finden und sich nicht unmittelbar ergeben. Die folgende Tabelle stellt den Metamodell-Komponenten jeweils die grafische Repräsentationsform gegenüber. Die Darstellung erfolgt in Anlehnung an [Holt01, S. 36f].

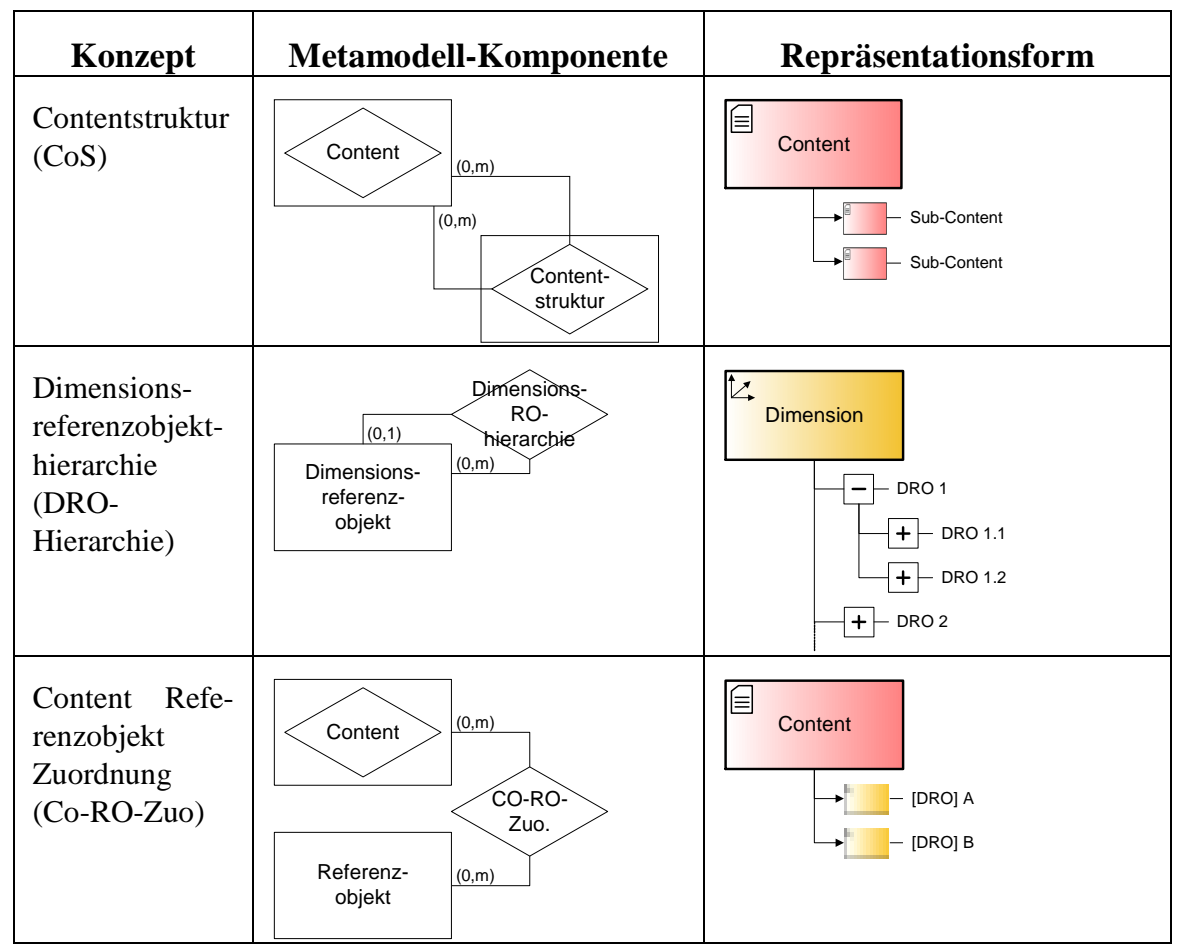




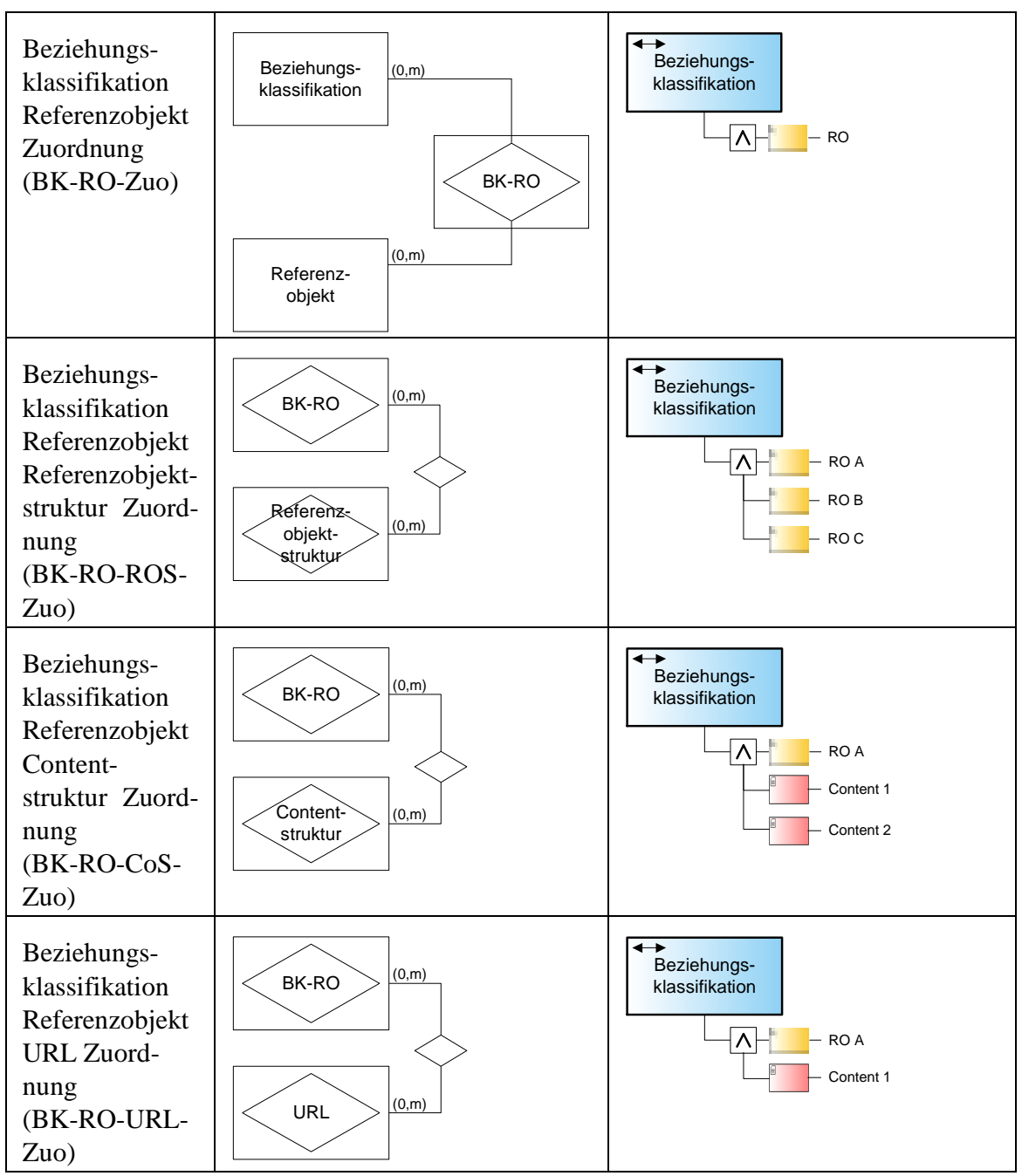

Tabelle 1: Notation der Sprache MetaWeb

\subsection{Anwendungsbeispiele der Technik MetaWeb}

Im Folgenden wird an Hand eines Beispielszenarios die Anwendung der Modellierungstechnik vorgestellt. Ein Handelsunternehmen strebt die Entwicklung eines WWW-basierten Intranets an, das als Wissensbasis für die Mitarbeiter dient. Inhalte der Wissensbasis sind z. B. Produkt- und Vertriebsinformationen, Aufgabenbeschreibungen, Nachrichten, Stellenausschreibungen etc. Die Realisierung der fachkonzeptionellen Spezifikation erfolgt in drei Schritten: 
Im ersten Schritt müssen Dimensionen identifiziert und die Dimensionsreferenzobjekte spezifiziert werden. Dadurch wird der für die jeweilige Organisation relevante Informationsraum aufgespannt. Für das Handelsunternehmen sind die Dimensionen Zeit, Region, Produkt und Organisation identifiziert worden. Dimensionsreferenzobjekte ermöglichen die Bildung von Hierarchien innerhalb von Dimensionen. Dadurch wird den späteren Anwendern die Navigation erleichtert, da diese sich an Hand von verbreiteten und akzeptierten Hierarchiestufen (z. B. Jahr, Quartal, Monat in der Dimension Zeit) im Informationssystem orientieren können. Innerhalb der identifizierten Dimensionen sind die in Abbildung 4 illustrierten Dimensionsreferenzobjekte festgelegt worden.
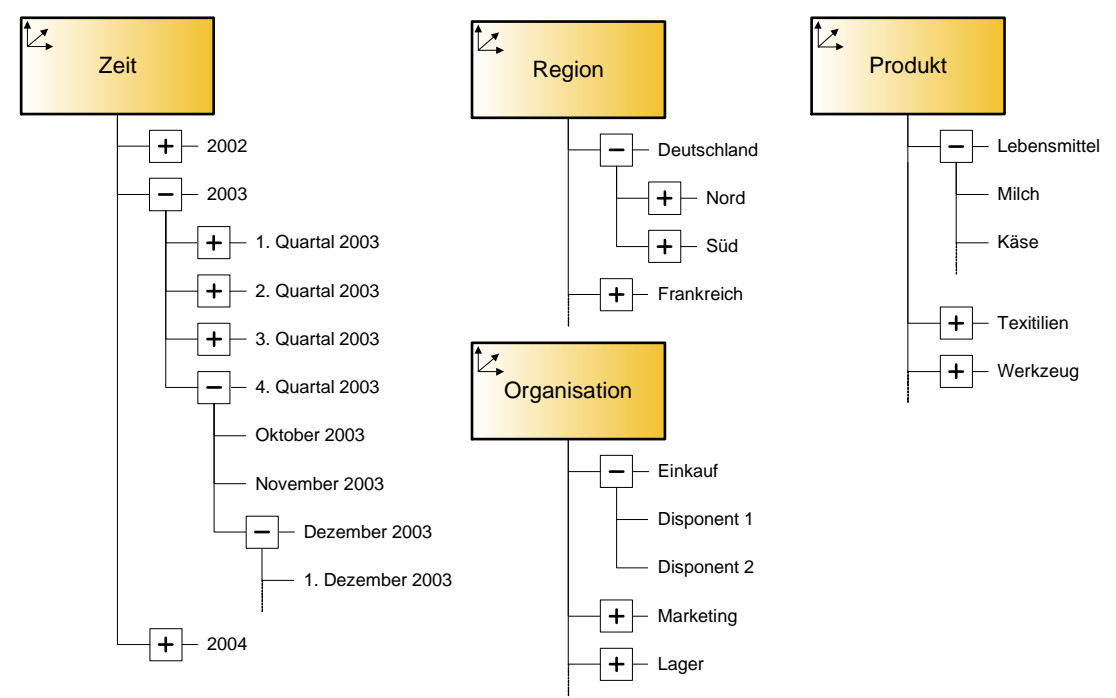

Abbildung 4: Modellierung von Dimensions-Referenzobjekt-Hierarchien

Im zweiten Schritt erfolgt die Platzierung des Contents an den entsprechenden Stellen im Informationsraum. Dazu wird jedem Content mindestens ein Referenzobjekt zugeordnet. Darüber hinaus muss eine eventuell vorhandene Contentstruktur abgebildet werden. 

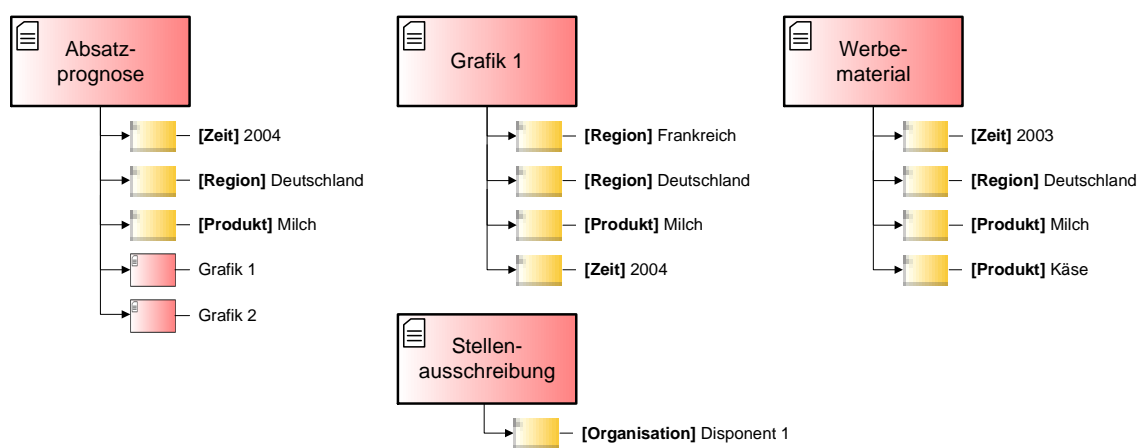

Abbildung 5: Modellierung und Zuweisung von Content

In der Abbildung 5 ist der Content Absatzprognose dargestellt, in dem die Verkaufszahlen für das Produkt Milch im Jahr 2004 in Deutschland prognostiziert werden. Dementsprechend ist dem Content das kombinierte Referenzobjekt ([Zeit] 2004, [Region] Deutschland, [Produkt] Milch) zugeordnet. Da über den Content Absatzprognose der Content Grafik 1 aufgerufen werden kann, ist diese dem Content Absatzprognose in Form einer Contentstruktur ebenfalls zugeordnet. Der untergeordnete Content Grafik 1 erbt die Referenzobjekte des Content Absatzprognose, kann aber zusätzliche Referenzobjekte enthalten. Es wird unterstellt, dass im Content Grafik 1 ein Vergleich zwischen der Absatzprognose des Produktes Milch in Deutschland und Frankreich in grafischer Form vorgenommen wird. Daher wird dem Content Grafik 1, neben den geerbten Referenzobjekten, zusätzlich das Dimensionsreferenzobjekt ([Region] Frankreich) zugeordnet. Weitere Beispiele für Content-Referenzobjekt-Zuordnungen (Co-RO-Zuo) sind der Content Werbematerial mit der Zuordnung zum kombiniertem Referenzobjekt ([Zeit] 2003, [Region] Deutschland, [Produkt] Milch, [Produkt] Käse) und der Content Stellenausschreibung, der dem Dimensionsreferenzobjekt ([Organisation] Disponent 1) zugeordnet ist.

In einem dritten Schritt werden Beziehungsklassifikationen identifiziert und modelliert. Damit eine semantische Navigation innerhalb des WWW-basierten Informationssystems ermöglicht wird, müssen mögliche Beziehungsarten von $\mathrm{Zu}$ ordnungen zwischen Content und Referenzobjekten festgelegt werden. Dabei können drei unterschiedliche Typen von Beziehungsklassifikationen unterschieden werden (vgl. Tabelle 1). Für das Handelsunternehmen sind die Beziehungsklassifikationen Verbundprodukt, Substitutionsprodukt und Zugriff exemplarisch modelliert (vgl. Abbildung 6).

Verbundprodukt und Substitutionsprodukt sind Beispiele für Beziehungsklassifikationen des Typs (BK-RO-ROS-Zuo). Die Dimensionsreferenzobjekte ([Produkt] Milch) und ([Produkt] Brot) sind der Beziehungsklassifikation Verbundprodukt zugeordnet. Dadurch wird ein semantischer Navigationspfad im Intranet realisiert, der einem Nutzer die Navigation von einem Content, in dem bspw. Informationen 
zu dem Produkt Brot aufgeführt sind, zu einem Content, der einen Bezug zum Produkt Milch enthält, ermöglicht.

,Zugriff' stellt ein Beispiel für die Beziehungsklassifikation des Typs (BK-ROURL-Zuo) dar. Über die Beziehungsklassifikation Zugriff wird es nur den Mitarbeitern der Organisationseinheit Einkauf erlaubt, auf den Content Absatzprognose zuzugreifen.

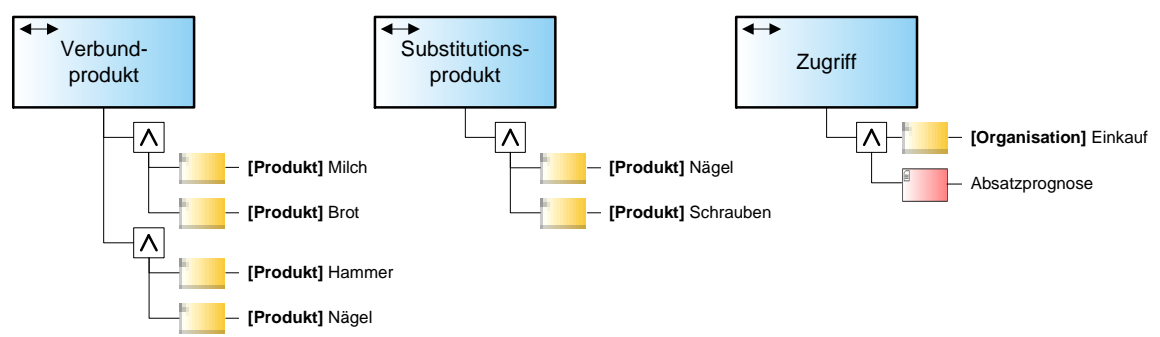

Abbildung 6: Modellierung und Zuweisung von Beziehungsklassifikationen

In den hier aufgeführten Beispielen, wird nicht immer ein Referenzobjekt zur Klassifikationen verwendet (der Platz rechts neben dem UND-Symbol ist leer). In diesen Fällen ist das Referenzobjekt leer (bzw. NULL).

Auf Basis der hier angedeuteten fachkonzeptionellen Spezifikation durch die Modellierungsmethode MetaWeb lassen sich im zu implementierenden WWWbasierten Informationssystem drei Klassen von Navigationsstrukturen realisieren.

Die erste Navigationsstruktur wird mit Hilfe der lediglich im Metamodell beschriebenen Navigationsknoten umgesetzt. Diese stellt die klassische, auf Rubriken basierende Navigationsart dar, die derzeitig in nahezu allen WWW-basierten Informationssystemen Anwendung findet. Beispiele für derartig gestaltete Menüeinträge sind Home, Mitarbeiter, Nachrichten, Produktinformationen, etc. Realisiert wird diese Navigationsart über speziell anwählbare URLs, die durch die Zuordnung eines Navigationsknotens zu einem Content entstehen. Diese Navigationsart kann bezogen auf das Anwendungsszenario z. B. genutzt werden, um von dem Content Absatzprognose über einen Navigationsknoten Mitarbeiter zu dem Content Stellenbeschreibung zu gelangen (vgl. Abbildung 5).

Die zweite Navigationsart stellt die Contentstruktur dar, die durch die Verknüpfung von verschiedenen miteinander in logischer Beziehung stehenden Contents realisiert wird. Ein Beispiel für eine Contentstruktur ist der Content Absatzprognose, dem der Content Grafik 1 zugeordnet ist.

Durch MetaWeb lässt sich eine weitere Klasse von Navigationsstrukturen realisieren, welche semantische Beziehungen zwischen Contents stärker zum Ausdruck bringt und somit über die herkömmlichen Navigationsstrukturen hinausgeht. Jeder sich im Informationssystem befindliche Content ist mindestens einem Referenzobjekt zugeordnet. Diese Referenzobjekte befinden sich in einem Informationsraum, 
der durch Dimensionen aufgespannt wird. Durch die Spezifikation der Dimensionen (hier Zeit, Region, Produkt, Organisation) wird der für das Handelsunternehmen relevante Informationsraum determiniert. Dadurch wird eine semantische Navigation zwischen Contents ermöglicht, die nicht explizit angegeben werden muss. So kann im Anwendungsszenario z. B. über den Content Absatzprognose 2003 zum Content Absatzprognose 2004 navigiert werden (vgl. dazu auch Abbildung 2).

Darüber hinaus ist es möglich, aus den modellierten Dimensionsreferenzobjekten ein zusätzliches Navigationsmenü abzuleiten, dass eine strukturierte Orientierung im Informationsraum ermöglicht. Dem Anwender kann ein baumartiges Menü zur Verfügung gestellt werden, an Hand dessen dieser Dimensionen ein- und ausblenden bzw. aufbrechen kann. Dadurch kann der Nutzer den Informationsraum aus verschiedenen Perspektiven betrachten und Informationsteilräume auswählen, zu denen er sich alle hier eingeordneten Inhalte anzeigen lassen kann. Derartige Teilräume sind im Metamodell in Kapitel 3 durch den Entitytyp Dimensionsausschnitt repräsentiert. Die Erstellung und Pflege eines solchen Navigationsmenüs ist, ein entsprechendes Modellierungswerkzeug vorausgesetzt, automatisiert möglich.

\section{Fazit und Ausblick}

WWW-basierte Informationssysteme sind zu einem festen Bestandteil vieler Informationssystem-Architekturen geworden. Niedrige Kosten, hohe Flexibilität und leistungsstarke Navigationsstrukturen sprechen für den Einsatz dieser Systeme zur Verwaltung semistrukturierter Daten. Trotz Verfügbarkeit der Basistechnologien (insbesondere XML und Web Ontologies) stellt die konzeptionelle Planung eines WWW-basierten Informationssystems nach wie vor eine große Herausforderung dar, an der heutige Projekte vielfach scheitern. Dies ist aus Sicht der Wirtschaftsinformatik insbesondere auf das Fehlen einer geeigneten Modellierungstechnik zurück zu führen, welche die Phase der fachkonzeptionellen Spezifikation in geeigneter Weise unterstützt.

Die Technik MetaWeb kann an dieser Stelle des Entwicklungsprozesses einen wertvollen Beitrag leisten. Durch die Abstraktion von implementierungstechnischen Details zeichnet sich MetaWeb durch eine leichte Verständlichkeit und Kommunizierbarkeit aus, die auch weniger technikaffine Fachanwender in die Lage versetzt komplexe WWW-basierte Informationssysteme fachkonzeptionell zu spezifizieren. Insbesondere die Verknüpfung von Inhalten mit Referenzobjekten, und die damit verbundene Erzeugung plausibler und interpretierbarer Navigationsstrukturen, ist geeignet, die Akzeptanz und Effizienz des Informationssystems zu erhöhen. Durch den systematischen, theoretisch fundierten Aufbau der Technik mit Hilfe des sprachkritischen Ansatzes wird sichergestellt, dass die Technik verständlich und flexibel erweiterbar ist. 
Zur Umsetzung in eine konkrete WWW-basierte Anwendung bietet sich die Unterstützung bzw. Konfiguration eines Content Management Systems durch die Methode an. Ebenso ist eine Weiterverarbeitung durch implementierungsnähere Modellierungsmethoden, wie Araneus, W3DT oder WebML denkbar, indem die mit MetaWeb erstellten Modelle automatisiert in die genannten Modellierungsmethoden überführt werden.

Weiterer Forschungsbedarf besteht hinsichtlich der Erweiterung der Technik. Zur korrekten Anwendung der Technik müssen die einzelnen Teilschritte im Rahmen der Handlungsanleitung genauer ausgeführt werden. Ferner kann die Technik gemäß dem Metamodell um weitere Sprachelemente erweitert werden, die zusätzliche Anforderungen realisieren. Zur Identifikation der zentralen Sprachelemente (etwa Dimensionen und Dimensionsreferenzobjekten) wird im Rahmen der weiteren Forschung die Eignung der Methoden der Informationsbedarfsanalyse untersucht werden.

\section{Literatur}

[Bec $\left.{ }^{+} 01\right]$ Becker, J.; Knackstedt, R.; Holten, R.; Hansmann, H.; Neumann, S.: Konstruktion von Methodiken: Vorschläge für eine begriffliche Grundlegung und domänenspezifische Anwendungsbeispiele. In: Becker, J. et al. (Hrsg.) Arbeitsberichte des Institutes für Wirtschaftsinformatik Nr. 77, 2001.

[Bec $\left.{ }^{+} 95\right]$ Becker, J.; Rosemann, M.; Schütte, R.: Grundsätze ordnungsmäßiger Modellierung. In: Wirtschaftsinformatik 37, 1995, S. 435-445.

[BeLa03] Benn, W.; Langer, O.: Semistrukturierte Datenmodelle und XML. In: Rahm, E.; Vossen, G. (Hrsg.) Web \& Datenbanken: Konzepte, Architekturen, Anwendungen. dpunkt-Verlag, Heidelberg, 2003, S. ${ }^{\circ 3-31 .}$

[Ber $\left.{ }^{+} 01\right]$ Berners-Lee, T. J.; Hendler, J.; Lassila, O.: The Semantic Web. http://www.sciam.com/article.cfm?articleID=00048144-10D2-1C7084A9809EC588EF21, 2001, Abruf am 2002-12-20.

[BeSc96] Becker, J.; Schütte, R.: Handelsinformationssysteme. Landsberg/Lech 1996.

[BiNu96] Bichler, M.; Nusser, S.: Modular Design of Complex Web-Applications with W3DT. Proceedings Workshop on Enabling Technology: Infrastructure for Colloborative Enterprises 5, 1996, S. 328-333.

[Bos $\left.{ }^{+} 98\right]$ Bos, B. et al.: Style Sheets Level 2 Spezification. http://www.w3.org/TR/RECCSS2/, 1998, Abruf am 2002-12-11.

[Can $\left.{ }^{+} 01\right]$ Candan, K. S.; Liu, H.; Suvarna, R.: Resource description framework: metadata and its applications. ACM SIGKDD Explorations Newsletter 3, 2001: S. 6-19. 
[Cer $\left.{ }^{+} 00\right]$ Ceri, S.; Fraternali, P.; Paraboschi, S.: Web Modeling Language (WebML): a modeling language for designing Web sites. Proceedings of the 9th International World Wide Web Conference, 2000, S. 137-157.

[Chen76] Chen, P. P. S.: The Entitiy Relationship Model - Towards a unified View of Data. ACM Transactions on Database Systems 1, 1976, S. 9-36.

[Cod $\left.{ }^{+} 93\right]$ Codd, E.F.; Codd, S.B.; Salley, C.T.: Providing OLAP to User-Analysts:An IT Mandate. White Paper. E.F. Codd Associates. 1993. http://www.unituebingen.de/wi/forschung/Arbeitsberichte(3)/ab_wi16.ok/ab_wi16.pdf. Abruf vom 06.02.2003.

[Ehle03] Ehlers, L. H.: Fachkonzeptionelle Spezifikation von Content Management Anwendungen. Dissertation, Universität Münster, 2003.

[FeSi01] Ferstl, O.; Sinz, E.: Grundlagen der Wirtschaftsinformatik. Band 1. OldenbourgVerlag, München, Wien 2001.

[Gar ${ }^{+}$93] Garzotto, F.; Paolini, P.; Schwabe, D.: HDM - A model-based approach to hypertext application design. ACM Transactions of Information Systems 11, S. 1-26.

[HaSc90] Halasz, F.; Schwartz, M.: The Dexter Hypertext Reference Model. Proceedings of the Hypertext Workshop, National Institute of Standards (NIST) Special Publication, 1990, S. 95-133.

[Holt01] Holten, R.: The MetaMIS Approach for the Specification of Management Views on Business Processes. In: Becker, J. et al. (Hrsg.) Arbeitsberichte des Institutes für Wirschaftsinformatik Nr. 84, 2001.

[Holt99] Holten, R.: Entwicklung von Führungsinformationssystemen: Ein methodenorientierter Ansatz. Dissertation, Universität Münster, 1999.

[Isa ${ }^{+}$95] Isakowitz, T.; Stohr, E.; Balasubramanian P.: RMM: a methodology for structured hypermedia design. Communications of the ACM 38, 1995, S. 34-44.

[KaLo73] Kamlah, W.; Lorenzen, P.: Logische Propädeutik. Mannheim, 1973.

[Krcm03] Krcmar, H.: Informationsmanagement. Springer-Verlag, Berlin et. al. 2003.

[LaSw99] Lassila, O; Swick, R. R.: Resource Description Framework (RDF) Model and Syntax Specification. http://www.w3.org/TR/1999/REC-rdf-syntax-19990222/, 1999, Abruf am 2003-01-12.

[Lehm99] Lehmann, F. R.: Normsprache. http://www.gi-ev.de/informatik/lexikon/inf-lexnormsprache.shtml, 1999, Abruf am 2003-02-10.

[Meri ${ }^{+}$03] Merialdo, P.; Atzeni, P.; Giansalvatore, M.: Design and Development of dataintensive web sites: The Araneus approach. ACM Transactions on Internet Technology (TOIT) 3, 2003, S. 49-92.

[Sch $\left.{ }^{+} 96\right]$ Schwabe, D.; Rossi, G.; Barbosa, S. D. J.: Systematic hypermedia application design with OOHDM. Proceedings of the 7th ACM Conference on Hypertext 7, 1996, S. 116-128. 
[Sche98] Scheer, A.-W.: Wirtschaftsinformatik: Referenzmodelle für industrielle Geschäftsprozesse, Berlin u. a., 1998.

[Seib01] Seibt, D.: Anwendungssystem. In: Mertens, P. et al. (Hrsg.) Lexikon der Wirtschaftsinformatik. Springer-Verlag, Berlin et. al. 2001.

[Smi $\left.{ }^{+} 02\right]$ Smith, M. K.; McGuiness, D.; Volz, R.; Welty, C.: Web Ontology Language (OWL) Guide Version 1.0. http://www.w3.org/TR/owl-guide/, 2002, Abruf am 200212-16.

[Strah96] Strahringer, S.: Metamodellierung als Instrument des Methodenvergleichs: Eine Evalulierung am Beispiel objektorientierter Analysemethoden. Dissertation, TH Darmstadt, 1996.

[Teub99] Teubner, A.: Organisations- und Informationssystemgestaltung. Theoretische Grundlagen und integrierte Methoden, Dissertation, Universität Münster, 1997. 